\title{
Neuroimaging Features of COVID-19: Retrospective Northern Italy Multicenter Study and a Scoping Review of the Prevalence of COVID-19 Associated Acute Cerebrovascular Diseases
}

\section{Andrea Giorgianni}

Department of Neuroradiology, Di Circolo e Fondazione Macchi Hospital, Varese

Francesco D'Amore ( $\square$ francesco.damore@asst-settelaghi.it )

Department of Neuroradiology, Di Circolo e Fondazione Macchi Hospital, Varese

Gabriele Vinacci

Division of Radiology, University of Insubria, Varese

\section{Edoardo Agosti}

Division of Neurosurgery, Department of Biotechnology and Life Sciences, University of Insubria, Varese

\section{Letterio Politi}

Neuroradiology Division, Humanitas Clinical and Research Center - IRCCS -, Rozzano

Andrea De Vito

UO Neuroradiology, San Gerardo Hospital, ASST Monza, Monza

\section{Alessandra Polistena}

UO Neuroradiology, San Gerardo Hospital, ASST Monza, Monza

\section{Luca Valvassori}

UO Neuroradiology, San Gerardo Hospital, ASST Monza, Monza

\section{Mirko Trentadue}

SC Neuroradiology, Carlo Poma Hospital, Mantova

\section{Lisa Nicoli}

UO Neuroradiology, San Gerardo Hospital, ASST Monza, Monza

\section{Carlo Sozzi}

SC Neuroradiology, Carlo Poma Hospital, Mantova

\section{Sergio Balbi}

Division of Neurosurgery, Department of Biotechnology and Life Sciences, University of Insubria, Varese Anna Mercuri

Department of Neuroradiology, Di Circolo e Fondazione Macchi Hospital, Varese

\section{Alberto Terrana}

Department of Neuroradiology, Di Circolo e Fondazione Macchi Hospital, Varese 
University of Milan and Neuroradiology Unit IRCCS Cà Granda Foundation, Maggiore Policlinico Hospital, Milan

\section{Casale Silvia}

Neuroradiology Unit, IRCCS Cà Granda Foundation, Maggiore Policlinico Hospital, Milan

\section{Nunzio Nuzzi}

Neuroradiology Division, Humanitas Clinical and Research Center - IRCCS -, Rozzano

\section{Francesco Asteggiano}

Neuroradiology Division, Humanitas Clinical and Research Center - IRCCS -, Rozzano

\section{Valentina Genovese}

U.O.C Radiology and Neuroradiology Diagnostic and Interventional, Policlinic IRCCS "San Matteo", Pavia

\section{Elvis Lafe}

U.O.C Radiology and Neuroradiology Diagnostic and Interventional, Policlinic IRCCS "San Matteo", Pavia

\section{Elena Bianchini}

U.O.C Radiology, Civile Di Legnano Hospital, ASST Ovest Milanese, Legnano

Pietro Bernasconi

U.O.C Radiology, Circolo di Busto Arsizio Hospital, ASST Valle Olona, Busto Arsizio

\section{Enrico Colli-Tibaldi}

Department of Radiology, Maggiore Hospital, ASST Lodi

\section{Laura Longhi}

U.O.C Radiology, S.Anna Como Hospital, ASST Lariana, Como

\section{Francesca Bandiera}

U.O. Radiology, S.Antonio Abate Hospital, ASST Valle Olona, Gallarate

\section{Fabio Baruzzi}

Department of Neuroradiology, Di Circolo e Fondazione Macchi Hospital, Varese

\section{Research Article}

Keywords: COVID-19, Coronavirus, Neuroimaging, Neurological, Cerebrovascular disease

Posted Date: January 29th, 2021

DOI: https://doi.org/10.21203/rs.3.rs-150229/v1

License: (c) (1) This work is licensed under a Creative Commons Attribution 4.0 International License. Read Full License 
Neuroimaging features of COVID-19: retrospective Northern Italy multicenter study and a scoping review of prevalence of COVID-19 associated acute cerebrovascular diseases

Giorgianni $A^{1}$, D’Amore $F^{1}$, Vinacci $G^{2}$, Agosti $E^{3}$, Politi $L S^{4}$, De Vito $A^{5}$, Polistena $A^{5}$, Valvassori $L^{5}$, Trentadue $M^{6}$, Nicoli $L^{6}$, Sozzi $C^{6}$, Balbi $S^{3}$, Mercuri $A^{1}$, Terrana $A V^{1}$, Triulzi $\mathrm{F}^{7}$, Casale $\mathrm{S}^{8}$, Nuzzi NP ${ }^{4}$, Asteggiano $\mathrm{F}^{4}$, Genovese $\mathrm{V}^{9}$, Lafe $\mathrm{E}^{9}$, Bianchini $\mathrm{E}^{10}$, Bernasconi $\mathrm{P}^{11}$, Colli-Tibaldi $\mathrm{E}^{12}$, Longhi $\mathrm{L}^{13}$, Bandiera $\mathrm{F}^{14}$, Baruzzi $\mathrm{F}^{1}$

1 Department of Neuroradiology, Di Circolo e Fondazione Macchi Hospital, Varese

${ }^{2}$ Division of Radiology, University of Insubria, Varese

${ }^{3}$ Division of Neurosurgery, Department of Biotechnology and Life Sciences, University of Insubria, Varese

${ }^{4}$ Neuroradiology Division, Humanitas Clinical and Research Center - IRCCS -, Rozzano

${ }^{5}$ UO Neuroradiology, San Gerardo Hospital, ASST Monza, Monza

${ }^{6}$ SC Neuroradiology, Carlo Poma Hospital, Mantova

7 University of Milan and Neuroradiology Unit IRCCS Cà Granda Foundation, Maggiore Policlinico Hospital, Milan

${ }^{8}$ Neuroradiology Unit, IRCCS Cà Granda Foundation, Maggiore Policlinico Hospital, Milan

9 U.O.C Radiology and Neuroradiology Diagnostic and Interventional, Policlinic IRCCS "San Matteo", Pavia

${ }^{10}$ U.O.C Radiology, Civile Di Legnano Hospital, ASST Ovest Milanese, Legnano

${ }^{11}$ U.O.C Radiology, Circolo di Busto Arsizio Hospital, ASST Valle Olona, Busto Arsizio

${ }^{12}$ Department of Radiology, Maggiore Hospital, ASST Lodi

Department of Radiology, Civico Hospital Codogno, ASST Lodi

${ }^{13}$ U.O.C Radiology, S.Anna Como Hospital, ASST Lariana, Como

${ }^{14}$ U.O. Radiology, S.Antonio Abate Hospital, ASST Valle Olona, Gallarate

\section{Corresponding Author:}

Francesco D'Amore,

Department of Neuroradiology, Di Circolo e Fondazione Macchi Hospital, Viale Luigi Borri, 57, Varese

21100, Italy

Email: neuroradiologia.varese@gmail.com 
Keywords COVID-19 - Coronavirus - Neuroimaging - Neurological - Cerebrovascular disease

\author{
Abbreviations \\ COVID-19 coronavirus disease 2019 \\ SARS-CoV-2 Severe acute respiratory syndrome coronavirus 2 \\ CVD Cerebrovascular disease \\ CT Computed tomography \\ MRI Magnetic resonance imaging
}

DSA Digital subtraction angiography

PRES Posterior reversible encephalopathy syndrome

AIS Acute ischemic stroke

ICH Intracranial hemorrhages

SAH Subarachnoid hemorrhages

SDH Chronic subdural hematomas

MS Multiple sclerosis

ADEM Acute disseminated encephalomyelitis

GBS Guillain-Barré syndrome

GCS Glasgow coma scale

CNS Central nervous system 


\begin{abstract}
Background The primary aim of this study was to provide additional data of neuroimaging features of coronavirus disease 2019 (COVID-19) in a large-scale population admitted in several northern Italy institutions. The secondary aim was to analyze acute cerebrovascular disease (CVD) prevalence in COVID-19.
\end{abstract}

Methods A database of confirmed COVID-19 hospitalized patients who developed acute neurological symptoms and underwent any neuroimaging was retrospectively gathered from twelve institutions based in Lombardy from February 21 st to July 10th. To assess the prevalence of CVD we conducted a scoping review following the PRISMA extension guidelines for scoping reviews. We searched PubMed/Medline, SCOPUS and EMBASE databases for peer-reviewed in-press or published studies from December to January 2021 reporting CVD in COVID-19 patients.

Results Out of 90 COVID-19 patients who were referred to neuroimaging, 78 (87\%) showed CVD, in particular 65 had acute ischemic strokes (AIS), 8 had intracerebral hemorrhages, 2 subarachnoid hemorrhages $(\mathrm{SAH})$ and 3 showed clinical and imaging findings in keeping with posterior reversible encephalopathy syndrome (PRES); 6 patients (7\%) showed clinical and imaging findings highly suggestive of encephalitis; 3 patients (3\%) showed demyelinating diseases: 1 case of MS progression, 1 case of newly diagnosed MS and 1 case of acute disseminated encephalomyelitis (ADEM); 2 cases (2\%) acuity of chronic subdural hematoma (cSDH); 1 patient (1\%) with Guillain Barré syndrome. In addiction two patients with CVD developed cauda polyradiculitis and tetraparesis.

In our scoping review out of 3275 studies, 24 satisfied the inclusion criteria: in a pooled total population of 136198 patients, the pooled prevalence of CVD was $0.9 \%$. In particular $0.8 \%$ of AIS and $0.1 \%$ of ICH and $0.003 \%$ of PRES.

Conclusions Our study shows a high prevalence of CVD among patients who developed acute neurological symptoms, which is in line with papers reporting data comparable to ours. The heterogeneity of clinical reports, however, constitutes a limitation when comparing our findings with those of the clinical papers. Nonetheless, CVD could be a frightening association with COVID-19, particularly in critically ill patients. Healthcare policymakers and clinicians should be prepared to a likely increase in workload and to rearrange the strategy of healthcare delivery. 


\section{Background}

Since the World Health Organization (WHO) declared coronavirus disease 2019 (COVID-19) a pandemic on March 11, [1] worldwide SARS-CoV-2 infections are still dramatically increasing with over 90 million total cases and roughly 2 million deaths on January 13, 2021 [2]. USA has been suffering the highest number of infections and deaths, closely followed by India and Brazil [2]. On February 21, the first Italian COVID-19 case was diagnosed in the northern region of Lombardy. From there the infection rapidly spread in the whole country, which became the second world epicenter of COVID-19 outbreak, after China [2]. Currently Europe is facing a second wave of the pandemics. In Italy a total of 2,3 million infections and almost 80.000 deaths have been reported so far [2]. A growing body of evidences is unveiling the multi-organ involvement [3] by SARS-CoV-2 rather than the sole respiratory and gastrointestinal systems manifestations as initially thought. In particular the nervous system involvement most commonly manifests as anosmia, ageusia and impaired consciousness [4-7], however acute cerebrovascular diseases (CVD), hereinafter comprising ischemic/hemorrhagic stroke and PRES (posterior reversible encephalopathy syndrome), seem to have a high share among other etiologies of acute neurological impairment. As of today few large scale studies on neurological manifestations in COVID-19 and their imaging findings have been published [9, 16, 18, 23-28], thus the vast majority of current literature consists of case series or single case reports and focus on a single neurological manifestation [8-22]

The aims of this study were to collect further data on neuroimaging features of COVID-19 patients and to estimate the prevalence of cerebrovascular disease in SARS-CoV-2 infection using, respectively, a multicenter retrospective study design and a scoping review of the literature.

\section{Material and Methods}

\section{Multicenter retrospective study}

\section{Study design}

We retrospectively collected imaging and clinical data from eleven major Lombard institutions: *BLINDED* in the time frame between February to July, including hospitalized patients with: 1) symptoms of SARS-CoV-2 infection and positive rRT-PCR; 2) concurrent or subsequent acute neurological symptoms; 3) receiving neuroimaging scans of the brain and/or spine.

\section{Data collection}


Demographic data, comorbidities, neurological findings were retrieved from electronic medical records for each patient by each participating institution (Table 1).

Table 1

\begin{tabular}{|c|c|c|}
\hline \multirow{2}{*}{$\begin{array}{l}\text { Demographic data } \\
\text { Mean age (SD) }\end{array}$} & \multicolumn{2}{|c|}{ Total subset $\mathrm{n}=90$} \\
\hline & $69 \pm 15$ & \\
\hline Male & 50 & $55 \%$ \\
\hline \multicolumn{3}{|l|}{ Neurological findings } \\
\hline Paresis & 38 & $42.7 \%$ \\
\hline Altered level of consciousness & 19 & $21.1 \%$ \\
\hline Aphasia & 14 & $15.5 \%$ \\
\hline Dysarthria & 10 & $11.1 \%$ \\
\hline Asthenia & 8 & $8.9 \%$ \\
\hline Seizures & 6 & $6.7 \%$ \\
\hline Visual field impairment & 5 & $5.6 \%$ \\
\hline Headache & 4 & $4.4 \%$ \\
\hline Drowsiness & 1 & $1.1 \%$ \\
\hline Tetraparesis & 2 & $2.2 \%$ \\
\hline Other & 18 & $20.2 \%$ \\
\hline \multicolumn{3}{|c|}{ Comorbidities and pre-existing conditions } \\
\hline Hypertension & 33 & $36.6 \%$ \\
\hline Diabetes & 16 & $17.9 \%$ \\
\hline Malignancy & 15 & $16.6 \%$ \\
\hline Dyslipidemia & 12 & $13.3 \%$ \\
\hline AF & 11 & $12.2 \%$ \\
\hline Cardiovascular intervention & 10 & $11.1 \%$ \\
\hline CAD & 9 & $10 \%$ \\
\hline Previous stroke & 8 & $8.9 \%$ \\
\hline COPD & 6 & $6.7 \%$ \\
\hline CKD & 6 & $6.7 \%$ \\
\hline Obesity & 5 & $5.6 \%$ \\
\hline Other & 31 & $34.4 \%$ \\
\hline None & 19 & $21.1 \%$ \\
\hline \multicolumn{3}{|l|}{ Deaths } \\
\hline Total & $10 / 90$ & $11 \%$ \\
\hline Ischemic stroke & $5 / 65$ & $8 \%$ \\
\hline $\mathrm{ICH}$ & $3 / 8$ & $38 \%$ \\
\hline$S A H$ & $0 / 2$ & 0 \\
\hline PRES & $0 / 3$ & 0 \\
\hline Acute on chronic SDH & $0 / 2$ & 0 \\
\hline Encephalitis & $2 / 6$ & $33 \%$ \\
\hline Demyelinating diseases & $0 / 4$ & 0 \\
\hline
\end{tabular}

\section{Image acquisition and analysis}

All imaging scans were performed using standard of care protocols. Head computed tomography (CT) with or without contrast scans was employed as the first imaging modality. Brain and spine magnetic resonance imaging (MRI) with or without contrast were acquired either on $1.5 \mathrm{~T}$ or 3T scanners. Digital subtraction angiography (DSA) was performed on neuroangiography suites with the aim to perform endovascular thrombectomy. Scans were initially read by neuroradiologists at their own Institution and then reviewed by coauthors by each Institutions.

\section{Scoping review}

The scoping review was carried out according to the Preferred Reporting Items for Systematic Reviews and Metanalyses extension for scoping reviews (PRISMA-ScR) guidelines. Eligibility criteria were: 1) peer-reviewed original research studies, editorials, review studies published or in- 
press, 2) population with confirmed diagnosis of COVID-19, 3) reported data on cerebrovascular diseases. Studies published in any language were considered eligible. Unpublished or ongoing studies and case reports were not included. Boolean logic was employed to search MEDLINE/Pubmed, SCOPUS and EMBASE databases from December 2019 to January 12, 2021 using the following terms: COVID-19 AND stroke, COVID-19 AND "hemorrhage*", COVID-19 AND "cereb*", COVID-19 AND cns, COVID-19 AND pres. Two authors independently searched and screened all results first by title and abstract to assess whether the studies fulfilled the inclusion criteria; included studies were further full text reviewed to retrieve data and pooled prevalence of CVD.

\section{Results}

\section{Multicenter retrospective study}

Out of a total of 7937 consecutive patients, 253 (3\%) patients had an abnormal neurological examination and underwent neuroimaging (Fig. 1).

Fig 1

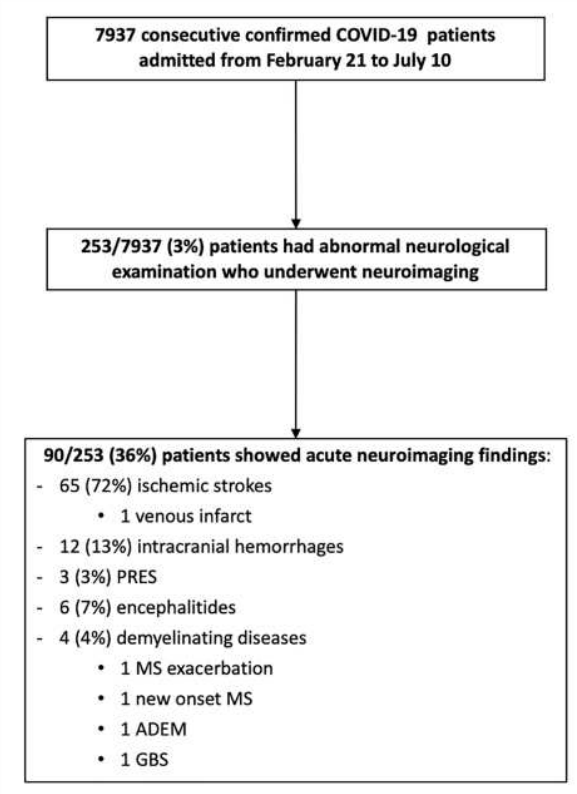

Of these, 90 (36\%) patients (55\% male predominance, mean age 69) showed acute neuroimaging findings. Eighty-eight (98\%) patients underwent brain CT, 41 (46\%) head and neck CT angiography (CTA), 32 (36\%) brain MRI, 5 (6\%) spinal MRI, 12 (13\%) had DSA (Table 2). 
Table 2

\begin{tabular}{|c|c|c|c|c|c|}
\hline & Head CT & Head and Neck CTA & Brain MRI & Spine MRI & DSA \\
& $88 / 90(98 \%)$ & $41 / 90(46 \%)$ & $32 / 90(36 \%)$ & $5 / 90(6 \%)$ & $12 / 90(13 \%)$ \\
\hline Ischemic stroke & $65 / 65(100 \%)$ & $35 / 65(54 \%)$ & $19 / 65(29 \%)$ & - & $12 / 65(18 \%)$ \\
\hline ICH & $8 / 8(100 \%)$ & $3 / 8(37 \%)$ & $3 / 8(37 \%)$ & $1 / 8(12 \%)$ & - \\
\hline SAH & $2 / 2(100 \%)$ & $0 / 2(0 \%)$ & $0 / 2(0 \%)$ & $1 / 2(50 \%)$ & - \\
\hline PRES & $3 / 3(100 \%)$ & $2 / 3(67 \%)$ & $3 / 3(100 \%)$ & - & - \\
\hline a/c SDH & $2 / 2(100 \%)$ & - & - & - & - \\
\hline Encephalitis & $6 / 6(100 \%)$ & $1 / 6(17 \%)$ & $5 / 6(83 \%)$ & - & - \\
\hline Demyelinating diseases & $2 / 4(50 \%)$ & $0 / 4(0 \%)$ & $2 / 4(50 \%)$ & $3 / 4(75 \%)$ & - \\
\hline
\end{tabular}

Eighty patients (89\%) had imaging findings of acute cerebrovascular diseases (Table 3), in particular, 65 patients suffered ischemic strokes, 12 patients had intracranial hemorrhages and 3 patients showed typical findings of posterior reversible encephalopathy syndrome (PRES), that was primarily hemorrhagic in 2 cases $(66 \%)$.

Table 3

\begin{tabular}{|c|l|}
\hline Neuroimaging findings & Notes \\
\hline Ischemic stroke & $\begin{array}{l}65 \% \text { involved anterior circulation, 23\% posterior circulation, 11\% } \\
\text { multifocal lesions, 1 venous stroke; 11\% developed hemorrhagic } \\
\text { transformation; 18\% elegible for endovascular treatment. }\end{array}$ \\
\hline ICH & $\begin{array}{l}50 \% \text { supra and infratentorial, 50\% supratentorial, 12.5\% basal } \\
\text { nuclei; 1 patient developed transient flaccid tetraparesis. }\end{array}$ \\
\hline SAH & $\begin{array}{l}100 \% \text { non traumatic and without underlying vascular } \\
\text { malfomations; } 1 \text { patient developed transient flaccid tetraparesis. }\end{array}$ \\
\hline PRES & 2 cases showed multiple hemorrhagic foci. \\
\hline a/c SDH & $100 \%$ non traumatic. \\
\hline Encephalitis & $\begin{array}{l}100 \% \text { involved the temporal lobes; } 1 \text { cerebellar peduncles } \\
\text { involved, 1 menigenal enhancement }\end{array}$ \\
\hline Demyelinating diseases & 1 MS exacerbation, 1 new onset MS, 1 ADEM, 1 GBS \\
\hline
\end{tabular}

Among the 65 cases of acute ischemic stroke (AIS), $42(65 \%)$ involved the anterior circulation (fig. 2), 15 (23\%) posterior circulation, 7 (11\%) were multifocal and 1 (2\%) was a venous stroke (fig. 3 ). 
Fig

2
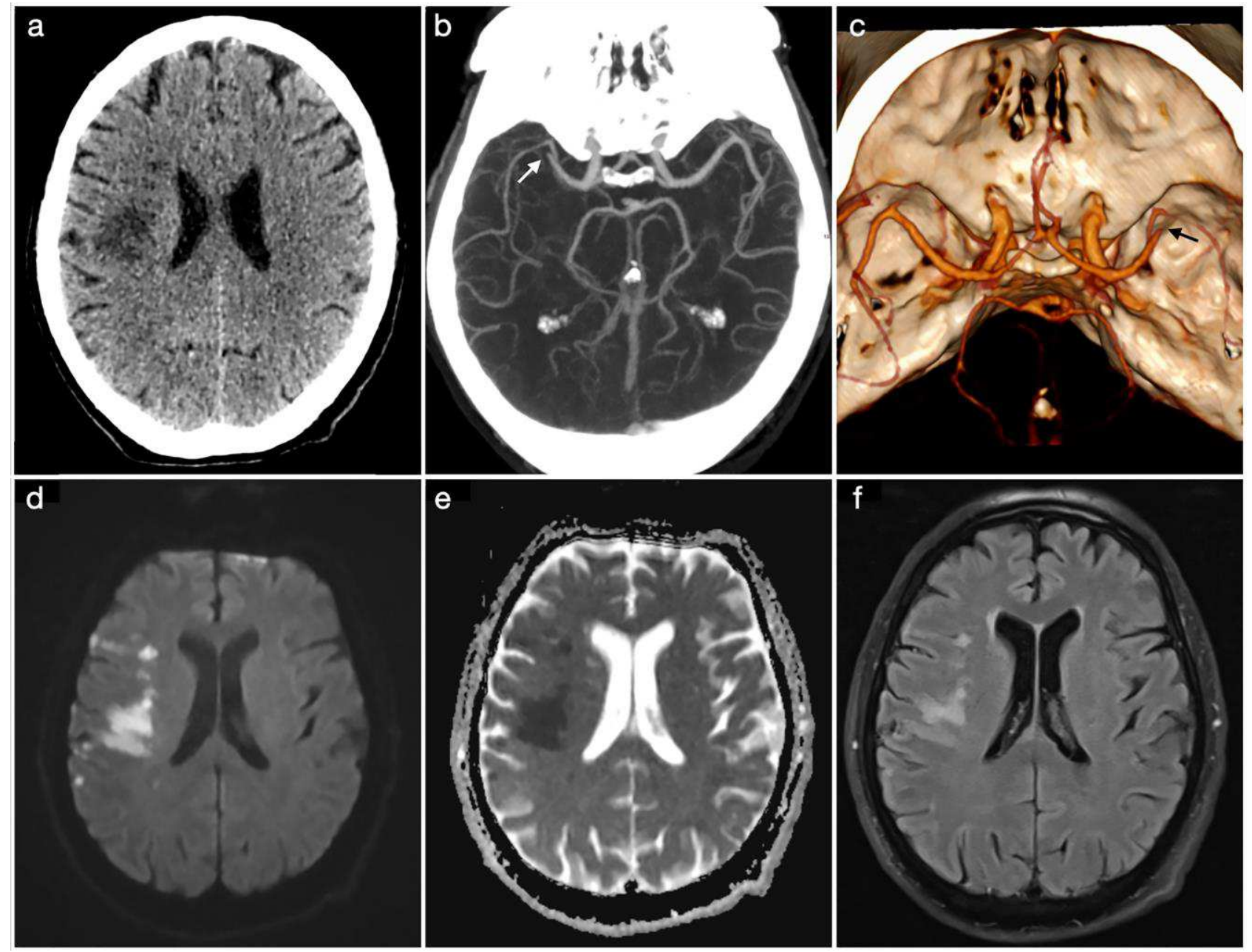
Fig 3

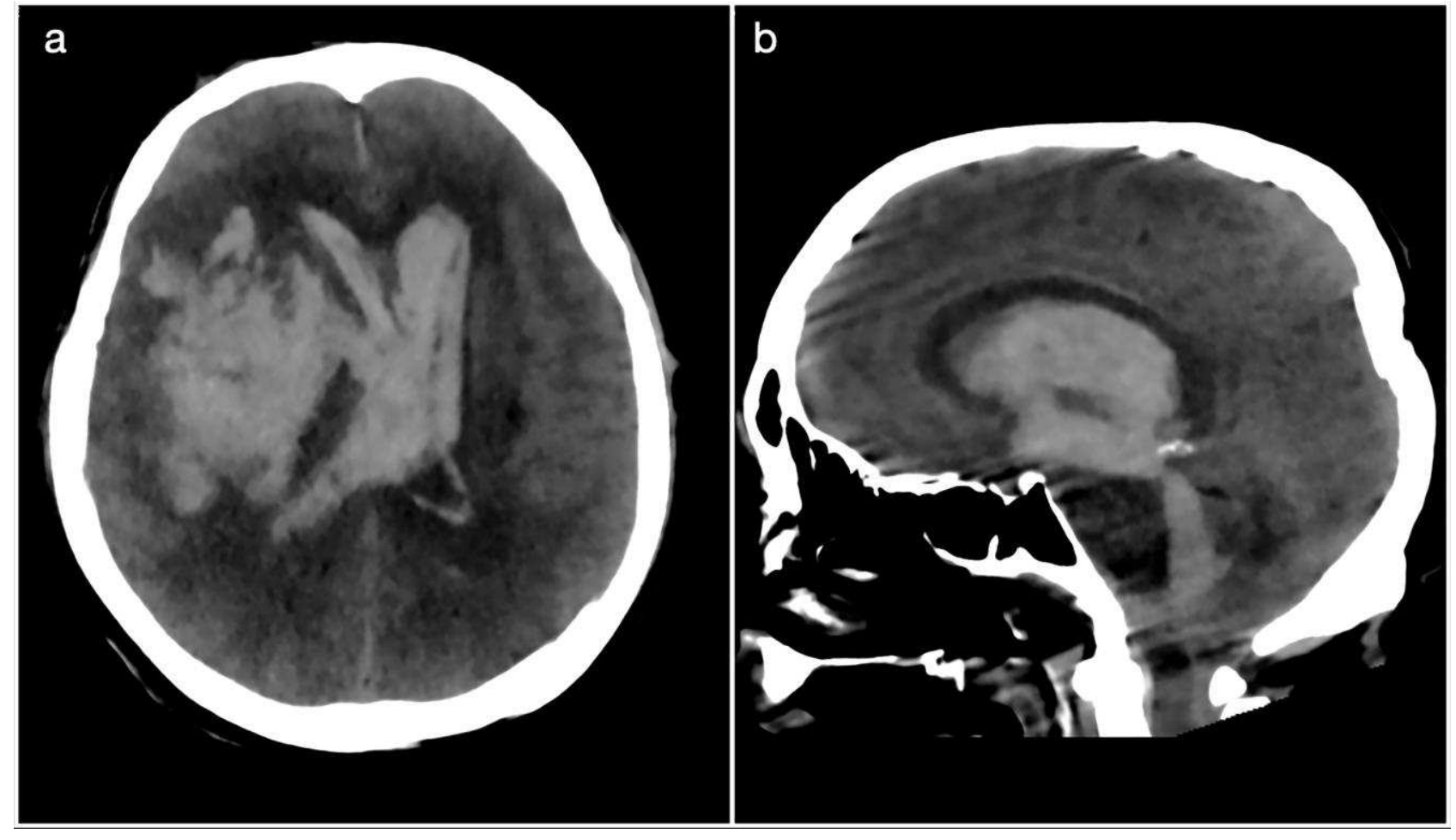

Of all intracranial hemorrhages (ICH), 8 were intraparenchymal (fig 4), 2 non-aneurysmal and nontraumatic subarachnoid hemorrhages (SAH) and 2 acute on chronic subdural hematomas (SDH). 
Fig 4
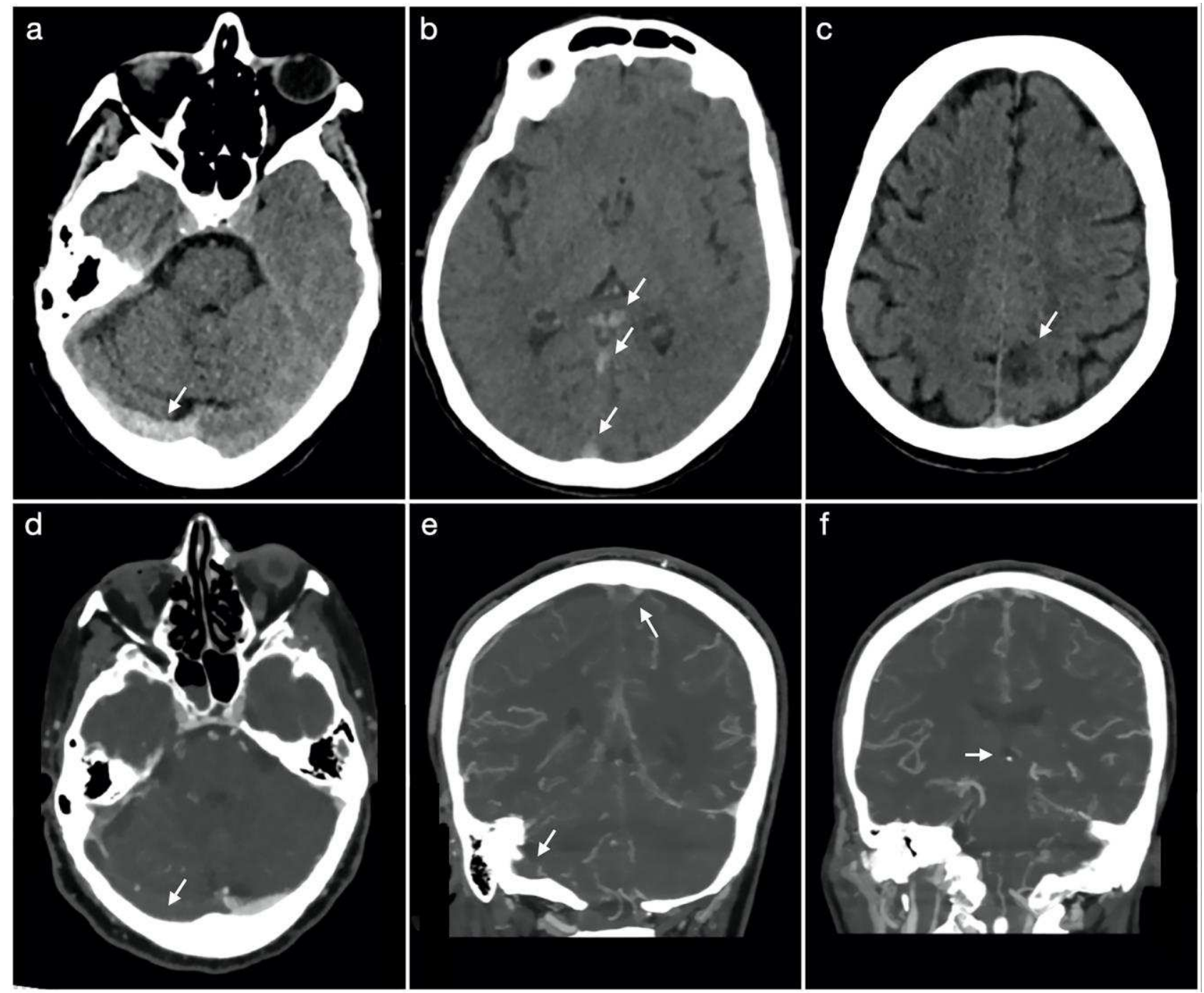

All 6 patients presenting with acute encephalopathy, showed temporal lobe lesions in keeping with encephalitis (Table 3). We also observed 4 (4\%) inflammatory demyelinating disorders, in particular 1 case of multiple sclerosis (MS) exacerbation, 1 case of newly diagnosed MS with supra-tentorial lesions, 1 case of acute disseminated encephalomyelitis (ADEM) with bilateral, multifocal lesions and 1 case of Guillain-Barré Syndrome (GBS). Three patients had spinal involvement: the above described GBS, 1 patient with SAH that developed polyradiculitis and transient flaccid tetraparesis, and 1 patient with a frontal hemorrhage that developed transient flaccid tetraparesis. The most frequent reported neurological symptoms were those related to acute stroke, followed by altered level of consciousness that was reported in 19 patients, of which, 16 had a Glasgow Coma Scale (GCS) score less than 7.

The highest mortality rate was related to ICH (38\%) followed by the encephalitis subgroup (33\%) (Table 1). Hypertension (36.6\%), diabetes (17.9\%) were the most frequent comorbidities, followed 
by previous or current history of malignancy (16.6\%). The most relevant comorbidities, neurological findings are summarized in Table 1. Prevalence data of our cohort are shown in Table 4.

Table 4

\begin{tabular}{|c|c|c|c|c|c|}
\hline & Cases $(n=90)$ & $\begin{array}{l}\text { Mean age } \\
\text { (Range) }\end{array}$ & Male & $\begin{array}{l}\text { COVID-19 } \\
(\mathrm{n}=7937)\end{array}$ & $\begin{array}{l}\text { COVID-19 with neurological } \\
\text { symptoms }(n=253)\end{array}$ \\
\hline CVD & 80 & 71 (21-90) & $59 \%$ & $1.01 \%$ & $31.6 \%$ \\
\hline AIS & 65 & $72(47-88)$ & $65 \%$ & $0.82 \%$ & $25.7 \%$ \\
\hline $\mathrm{ICH}$ & 8 & $60(21-84)$ & $50 \%$ & $0.10 \%$ & $3.2 \%$ \\
\hline$S A H$ & 2 & $84(78-90)$ & $100 \%$ & $0.03 \%$ & $0.8 \%$ \\
\hline PRES & 3 & $70(63-84)$ & $100 \%$ & $0.04 \%$ & $1.2 \%$ \\
\hline$a / c S D H$ & 2 & $67(49-85)$ & $50 \%$ & $0.03 \%$ & $0.8 \%$ \\
\hline Encephalitis & 6 & $69(40-90)$ & $83 \%$ & $0.08 \%$ & $2.4 \%$ \\
\hline $\begin{array}{l}\text { Demyelinating } \\
\text { diseases }\end{array}$ & 4 & $43(9-65)$ & $50 \%$ & $0.05 \%$ & $1.6 \%$ \\
\hline Total & 90 & 69 (9-90) & $55 \%$ & $1.13 \%$ & $35.6 \%$ \\
\hline
\end{tabular}

CVD was the commonest finding in patients who underwent neuroimaging (32\%, 80/253), followed by encephalitis $(2.4 \%, 6 / 253)$. Among CVD, AIS occurred more frequently $(26 \%, 65 / 253)$. In the total COVID-19 population, CVD prevalence was $1 \%$ (Table 4).

\section{Scoping review}

Titles and abstracts of 1643 studies were reviewed after excluding $n=1632$ duplicates out of a total 3275 records: 726 studies met the inclusion criteria (Fig. 5) and were full text screened.

Fig 5 


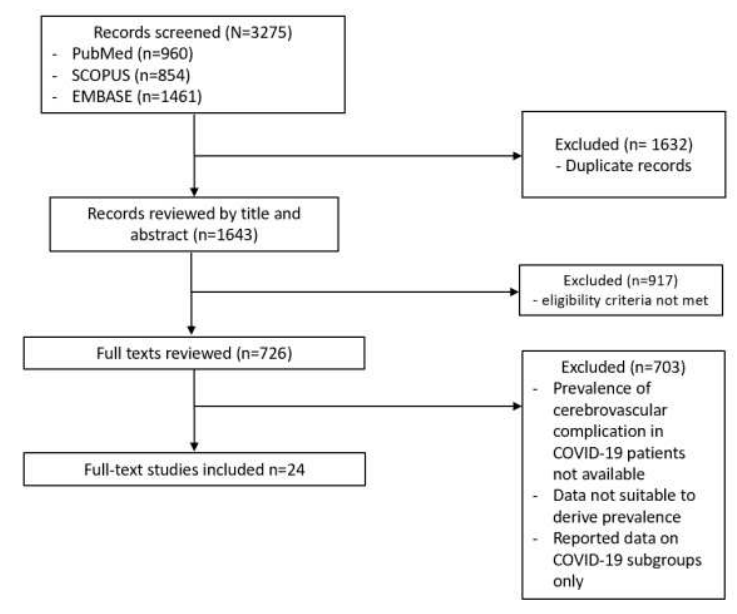

Of these, 24 studies [4, 27, 29-50] showed appropriate data to derive prevalence: thirteen retrospective multicentric studies, and eleven retrospective single center studies (Table 5), from the North America, Europe, Asia and Oceania.

Table 5

\begin{tabular}{|c|c|c|c|c|c|c|c|c|c|c|c|c|c|c|}
\hline \multirow{3}{*}{\begin{tabular}{|l|} 
Authors \\
Mao et al ${ }^{4}$
\end{tabular}} & \multirow{3}{*}{$\begin{array}{c}\begin{array}{c}\text { Date of } \\
\text { Publication }\end{array} \\
10-\text { Apr }\end{array}$} & \multirow{3}{*}{$\begin{array}{c}\text { Study design } \\
\text { Retrospective } \\
\text { multicentric }\end{array}$} & \multirow{3}{*}{$\begin{array}{l}\text { Country } \\
\text { China, Wuhan }\end{array}$} & \multirow{3}{*}{$\begin{array}{c}\begin{array}{c}\text { Population of } \\
\text { COVID-19 }\end{array} \\
214\end{array}$} & \multirow{2}{*}{\multicolumn{2}{|c|}{ Age $^{*}$}} & \multirow{3}{*}{$\begin{array}{l}\text { Male } \\
41 \%\end{array}$} & \multirow{3}{*}{$\begin{array}{c}\text { Cohort of CovID-19 with } \\
\text { neurological symptoms } \\
78(36.4 \%)\end{array}$} & \multicolumn{3}{|c|}{ CVD in COVID-19 } & \multicolumn{3}{|c|}{ CVD in COVID-19 with neurological symptoms } \\
\hline & & & & & & & & & AIS & $\mathrm{ICH}$ & PRES & Als & $\mathrm{ICH}$ & PRES \\
\hline & & & & & 52.7 & 15.5 & & & $5(2.3 \%)$ & $1(0.5 \%)$ & - & $6.4 \%$ & $1.3 \%$ & - \\
\hline${ }_{29}^{(1)}$ Lodigiani et al & 23-Apr & $\begin{array}{l}\text { Retrospective } \\
\text { single center }\end{array}$ & Italy, Milan & 388 & 66 & (55-75) & $68 \%$ & Not specified & $9(2.4 \%)$ & - & - & - & - & - \\
\hline Giorgianni et al ${ }^{27}$ & 15-May & $\begin{array}{l}\text { Retrospective } \\
\text { single center }\end{array}$ & Italy, Varese & 1000 & $71^{* * *}$ & $\begin{array}{l}(21 . \cdot \\
88)^{+* *}\end{array}$ & $46 \%^{* * *}$ & $26(2.6 \%)$ & $4(0.4 \%)$ & $5(0.5 \%)$ & - & $15.4 \%$ & $19.2 \%$ & - \\
\hline Jain et a ${ }^{30}$ & 19-May & $\begin{array}{l}\text { Retrospective } \\
\text { single center }\end{array}$ & USA, New York & 3218 & $64^{* * *}$ & $\begin{array}{l}(2 \text { weeks- } \\
105 \\
\text { year) }\end{array}$ & $60.7 \%$ & $454(14.1 \%)$ & $26(0.8 \%)$ & $9(0.2 \%)$ & - & $5.7 \%$ & $1.9 \%$ & - \\
\hline Mahammedi et al ${ }^{31}$ & 21-May & $\begin{array}{l}\text { Retrospective } \\
\text { multicentric }\end{array}$ & $\begin{array}{l}\text { Italy, Brescia, } \\
\text { Novara, Sassari }\end{array}$ & 725 & $69^{* *}$ & $15^{* *}$ & $64 \% \%^{* *}$ & $108(14.9 \%)$ & $34(4.6 \%)$ & $6(0.8 \%)$ & $1(0.1 \%)$ & $31.5 \%$ & $5.5 \%$ & $0.9 \%$ \\
\hline (2) Dogra et al ${ }^{32}$ & 23-May & $\begin{array}{l}\text { Retrospective } \\
\text { multicentric }\end{array}$ & USA, New York & 3824 & $62^{* *}$ & $\begin{array}{l}(37- \\
83)^{*+}\end{array}$ & $79 \% * *$ & $755(19.7 \%)$ & - & $33(0.9 \%)$ & - & - & $4.4 \%$ & - \\
\hline (1) Yaghi et al. ${ }^{33}$ & 26-May & $\begin{array}{l}\text { Retrospective } \\
\text { multicentric }\end{array}$ & USA, New York & 3556 & $62.5^{* *}$ & $17^{* *}$ & $72 \% *$ & Not specified & $32(0.9 \%)$ & - & - & - & - & - \\
\hline${ }_{34}^{(1)}$ Cantador et al & 9-Jun & $\begin{array}{l}\text { Retrospective } \\
\text { single center }\end{array}$ & Spain, Madrid & 1419 & $76^{* *}$ & $7^{* *}$ & $77 \% \%^{* *}$ & Not specified & $8(0.5 \%)$ & - & - & - & - & - \\
\hline Pons-Esconda ${ }^{35}$ & 11-Jun & $\begin{array}{l}\text { Retrospective } \\
\text { single center }\end{array}$ & $\begin{array}{l}\text { Spain, } \\
\text { Barcellona }\end{array}$ & 2249 & $74^{* * *}$ & $\begin{array}{l}(50.2 . \\
90)^{*+*}\end{array}$ & $61 \%^{* * *}$ & $103(4.6 \%)$ & $13(0.6 \%)$ & $7(0.3 \%)$ & & $12.6 \%$ & $6.8 \%$ & \\
\hline Li et al. ${ }^{36}$ & 12-Jun & $\begin{array}{l}\text { Retrospective } \\
\text { single center }\end{array}$ & China, Huazhong & 219 & $75.7^{* *}$ & $10.8^{* *}$ & $56 \% *$ & $83(37.8 \%)$ & $10(4.6 \%)$ & $1(0.5 \%)$ & - & $12 \%$ & $1.2 \%$ & - \\
\hline Nalleballe et $\mathrm{al}^{37}$ & 17-Jun & $\begin{array}{l}\text { Retrospective } \\
\text { multicentric }\end{array}$ & $\begin{array}{l}\text { Global clinical } \\
\text { research } \\
\text { platform }\end{array}$ & 40469 & $\begin{array}{c}\text { not } \\
\text { specifie } \\
d\end{array}$ & $\begin{array}{c}\text { not } \\
\text { specifie } \\
d\end{array}$ & $45 \%$ & $9086(22.5 \%)$ & $406(1 \%)$ & & - & $4.5 \%$ & - & - \\
\hline (1) Merkler et al ${ }^{38}$ & 2-Jul & $\begin{array}{l}\text { Retrospective } \\
\text { multicentric }\end{array}$ & USA, New York & 1916 & $69^{* *}$ & $\begin{array}{l}(66- \\
78)^{* *}\end{array}$ & $58 \% * *$ & Not specified & $31(1.6 \%)$ & - & - & - & - & - \\
\hline Rothstein et al ${ }^{39}$ & 20-Jul & $\begin{array}{c}\text { Retrospective } \\
\text { multicentric }\end{array}$ & $\begin{array}{l}\text { USA, } \\
\text { Philadelphia }\end{array}$ & 844 & 59 & 18 & $48 \%$ & $209(24.8 \%)$ & $20(2.4 \%)$ & $8(0.9 \%)$ & . & $9.6 \%$ & $3.8 \%$ & . \\
\hline (1) Altschul et al ${ }^{40}$ & 26-Jul & $\begin{array}{l}\text { Retrospective } \\
\text { single center }\end{array}$ & USA, New York & 5227 & $67^{* *}$ & $15.5^{5^{* *}}$ & $60 \% *$ & Not specified & & $16(0.3 \%)$ & & - & . & \\
\hline Requena et al ${ }^{41}$ & 5-Aug & $\begin{array}{l}\text { Retrospective } \\
\text { single center }\end{array}$ & $\begin{array}{l}\text { Spain, } \\
\text { Barcellona }\end{array}$ & 2050 & $66.5^{* *}$ & $15.2^{2 *}$ & $56 \% * *$ & Not specified & $18(0.9 \%)$ & $4(0.2 \%)$ & $3(0.1 \%)$ & - & - & . \\
\hline Katz et al ${ }^{42}$ & 6-Aug & $\begin{array}{l}\text { Retrospective } \\
\text { multicentric }\end{array}$ & USA, New York & 10596 & $67.9^{* *}$ & $\begin{array}{l}(25- \\
94)^{* *}\end{array}$ & $55.8 \% \%^{* *}$ & Not specified & $72(0.7 \%)$ & $14(0.1 \%)$ & & . & . & \\
\hline Shahjouei et al ${ }^{43}$ & 17-Aug & $\begin{array}{c}\text { Retrospective } \\
\text { multicentric }\end{array}$ & $\begin{array}{l}\text { USA, Brazil, } \\
\text { Greece, } \\
\text { Ittaly, Finland, } \\
\text { Turkey, } \\
\text { Lebanon, Iran, } \\
\text { India, New } \\
\text { Zealand }\end{array}$ & 17799 & $66 *$ & $15^{* *}$ & $58 \% * *$ & Not specified & $123(0.7 \%)$ & $25(0.1 \%)$ & - & - & - & - \\
\hline IItaf et al ${ }^{44}$ & 18-Aug & $\begin{array}{l}\text { Retrospective } \\
\text { single center }\end{array}$ & $\begin{array}{l}\text { Pakistan, } \\
\text { Karachi }\end{array}$ & 350 & 49.5 & 17.4 & $70 \%$ & $68(19.4 \%)$ & $2(0.6 \%)$ & - & - & $3.9 \%$ & - & - \\
\hline $\begin{array}{l}\text { Romero-Sanchez } \\
\text { et a al }\end{array}$ & 25-Aug & $\begin{array}{l}\text { Retrospective } \\
\text { multicentric }\end{array}$ & Spain, Albacete & 841 & 66.4 & 14.96 & $56.2 \%$ & Not specified & $11(1.3 \%)$ & $3(0.4 \%)$ & - & - & - & - \\
\hline Xiong et al ${ }^{46}$ & 15-Sep & $\begin{array}{l}\text { Retrospective } \\
\text { multicentric }\end{array}$ & $\begin{array}{l}\text { China, Wuhan, } \\
\text { Chongqing, } \\
\text { Sichuan }\end{array}$ & 917 & 48.7 & 17.1 & $55 \%$ & $39(4.3 \%)$ & $10(1.1 \%)$ & - & & $25.6 \%$ & - & \\
\hline Siegler et a ${ }^{177}$ & 30-Sep & $\begin{array}{c}\text { Retrospective } \\
\text { multicentric }\end{array}$ & $\begin{array}{l}\text { USA, Spain, } \\
\text { Egypt, Romania }\end{array}$ & 14483 & $\begin{array}{c}\text { not } \\
\text { speciiie } \\
d\end{array}$ & $\begin{array}{c}\text { not } \\
\text { specifie } \\
d\end{array}$ & not specified & Not specified & $156(1.1 \%)$ & $28(0.2 \%)$ & - & - & - & - \\
\hline Rifino et al ${ }^{48}$ & 07-Oct & $\begin{array}{l}\text { Retrospective } \\
\text { single center }\end{array}$ & Italy, Bergamo & 1760 & $649^{* * *+}$ & $14.0^{*+* *}$ & $66 \% \%^{* * *}$ & $137(7.8 \%)$ & $37(2.1 \%)$ & $11(0.6 \%)$ & - & $27 \%$ & $8 \%$ & - \\
\hline Sabayan et al ${ }^{49}$ & 6-Nov & $\begin{array}{l}\text { Retrospective } \\
\text { multicentric }\end{array}$ & Iran & 18407 & $65^{* *}$ & $\begin{array}{l}(38- \\
93)^{* *}\end{array}$ & $79 \%{ }^{* *}$ & Not specified & $14(0.1 \%)$ & - & - & - & - & - \\
\hline (2) Lee et al ${ }^{50}$ & 11-Jan 2021 & $\begin{array}{l}\text { Retrospective } \\
\text { single center }\end{array}$ & USA, Hartford & 3727 & 62 & $(52-74)$ & $54 \%$ & Not specified & $6(0.2 \%)$ & & - & - & - & - \\
\hline \multirow[t]{2}{*}{ Total } & & & & 136198 & 64.8 & _. & $60.2 \%$ & $11146(8.2 \%)$ & \multicolumn{3}{|c|}{$\mathrm{n}: 1222(0.9 \%, 0.1-5.7 \%)$} & \multicolumn{3}{|c|}{$11 \%$} \\
\hline & & & & & & & & & $1047(0.8 \%)$ & $171(0.1 \%)$ & $4(0.003 \%)$ & $9.4 \%$ & $1.5 \%$ & $0.03 \%$ \\
\hline \multirow{3}{*}{\multicolumn{15}{|c|}{$\begin{array}{l}\text { (1) study cohort of ischemic strokes } \\
\text { (2) study cohort of intracranial hemorrage }\end{array}$}} \\
\hline & & & & & & & & & & & & & & \\
\hline \multirow{2}{*}{\multicolumn{15}{|c|}{ (2) study cohort of intracranial hemorrage }} \\
\hline & & & & & & & & & & & & & & \\
\hline
\end{tabular}

A total population of 136198 COVID-19 patients was included (60.2\% of males, mean age 64.8). Pooled prevalence of CVD was $0.9 \%$ (range $0.1-5.7 \%$ ), and ischemic stroke was the most common 
manifestation (1047/1222, 86\%). The prevalence of CVD in the cohort of patients with neurological symptoms $(\mathrm{n}=11146)$ was $11 \%$, based on twelve of the total nine studies since in the remainder that cohort was not specified [29, 33, 34, 38, 40-43, 47, 49, 50].

\section{Discussion}

In this study we showed neurological and imaging findings in consecutive COVID-19 patients admitted in eleven centers in Lombardy region, which was the first in Europe to bear the burden of the outbreak of COVID-19 epidemic. Acute CVD had the highest prevalence among the COVID-19 related acute neurological diseases. The association between acute cerebrovascular events and SARSCoV-2 infection has been reported in several studies, with ischemic events outnumbering the primarily hemorrhagic. This evidence has been ascribed to the neurotrophic and neuroinvasive tendency of SARS-CoV-2, specifically, to its interaction with ACE-2 host receptors expressed on neurons and nervous system endothelial cells membranes [51] which results in endothelial damage. [51-52]. Once it reaches the central nervous system (CNS), SARS-CoV-2 can determine the activation of self-reinforcing inflammatory response through a 'cytokine storm', causing irreversible neuronal damage [53]. In addition, the endothelial ruptures in cerebral capillaries, due to the inflammatory process, can contribute to the pathophysiology of SARS-CoV-2 brain damage [51]. Ischemic stroke, cerebral venous thrombosis included, could be related both to the development of endotheliitis and hypercoagulability status. In this regard, Spiezia et al. [54] described a severe hypercoagulability status related to the inflammatory response and Zhang et al. [55] found the presence of antiphospholipid antibodies in few patients. In addition, SARS-CoV-2 infection can lead to cardiovascular complications including incident atrial fibrillation [56-58], which in turn is a risk factor of to cardio-embolic cerebral infarction. In our cohort, the majority of CVD occurred in older patients (mean age: 71 year old) with typical risk factors for CVD, in accordance to other reports [38]. In addition, acute viral infections may increase the risk of ischemic stroke as noted by some authors [59]. It could therefore be speculated that SARS-Cov2 could possibly play a role as a precipitating factor in the development of CVD through diverse mechanisms. CVD has also been reported in other Coronavirus infections, as in MERS and SARS, even if most of the paper published were case series [60]. It is suggested that Coronavirus infections, and other respiratory infections, is an independent risk factor for acute cerebrovascular disease [61].

We had severe PRES cases, some of which were primarily hemorrhagic. The occurrence of PRES in COVID-19 has been reported by some [14, 21, 62-65] and, notably, a number cases were complicated by intracranial hemorrhages $[21,62,64,65]$. This evidence may support that endothelium 
inflammation and the resulting abnormal vasoconstriction has a role in the pathophysiology of PRES in COVID-19 patients [66]. Many studies have shown that COVID-19 effects on CNS and peripheral nervous system most often become apparent [4-7, 67] as anosmia, ageusia, impaired consciousness, dizziness and headache, on the other hand, acute CVD syndromes are less frequent but bear potentially permanent CNS dysfunction hence worse prognosis. Notably, compared to influenza virus, COVID-19 patients have higher prevalence of AIS, highlighting how COVID-19 may be a risk factor for AIS [38]. Furthermore Merkler et al. found that initial plasma D-dimer levels were higher in COVID-19 ischemic stroke versus patients with influenza [38]. The prevalence of acute CVD in COVID-19 population was $1.0 \%$ and on COVID-19 with neurological symptoms was $36 \%$, the highest among other neurological syndromes, which is comparable to the results in the pooled population from the scoping review (1\% vs $0.9 \%$ ). Compared to the Italian population, where the most recent prevalence data of CVD in the general population is $6.5 \%$ [68], according to our results, CVD was lower in COVID-19 patients. This could be due to the clinical setting, i.e. intensive care unit patients with multiorgan failure and to the difficulty to obtain a complete neurological examination (intubated patients) which may have led to an underestimation of the true prevalence.

Central nervous system damage associated with SARS-CoV-2 invasive potential may underly the development of encephalitis and myelitis. This evidence confirms the neurotrophic and neuroinvasive tendency of SARS-CoV-2 ACE-2 host receptor mediated expressed on brain and spinal cord neurons [51, 69, 70]. Only few case reports recently described the association of COVID-19 with demyelinating diseases [71]. However a clear causative correlation between SARS-CoV-2 and the new onset or exacerbation of demyelinating diseases is yet to be determined [72]. It has been speculated that SARS-CoV-2 may activate lymphocytes and induce an inflammatory response leading to exacerbation or new onset of demyelinating disorders [73, 74].

Although our study has one of the largest populations of COVID-19 patients with neurological manifestations and positive neuroimaging, further data and larger samples could widen further the multifaceted nervous system involvement in COVID-19. Our scoping review is limited by the heterogeneity of study designs of the included works, their retrospective nature, fragmentary data reported and relatively small samples.

\section{Conclusions}

Our multicenter retrospective observational data confirm the high variability of neuroimaging features of COVID-19, additionally, CVD has the highest prevalence among other acute neurological manifestations in our cohort as well as in the current literature. This evidence demands awareness 
among clinician and healthcare policy maker to hone the daily practice and healthcare delivery strategy towards a more efficient response to the pandemic.

\section{Declaration}

Ethics approval and consent to participate

Ethical approval for this study was waived by the ethics committee of the Di Circolo e Fondazione Macchi Hospital, ASST Settelaghi, Varese, Italy because the emergency setting and the retrospective nature of the study.

All methods were carried out in accordance with ethical committee guidelines and regulations.

Consent for publication

Written informed consent was obtained from all subjects before the study

Availability of data and materials

The datasets used and analyzed during the current study are available from the corresponding author on reasonable request.

Competing interests

Not applicable

Funding

KARDIA SRL sustained the submission fee, but KARDIA SRL had no role in the design of the study, neither in the collection, analysis, and interpretation of data nor in writing of the manuscript.

Authors' contributions

GA, DAF, VG, AE, PLS, DVA, PA, VL, TM, NL, SC, BS, MA, TAV, TF, CS, NNP, AF, GV, LE, BE, BP, C-TE, LL, BF and BF collected patients data. DAF, VG, AE and GA analyzed and interpreted the patient data. DAF and VG wrote the manuscript. DAF, VG, GA and LP review the manuscript. All authors read and approved the final manuscript

Acknowledgements

Not applicable.

\section{References}

1. WHO Director-General's opening remarks at the media briefing on COVID-19 - 11 March 2020. Available from: https://www.who.int/dg/speeches/detail/who-director-general-sopening-remarks-at-the-media-briefing-on-covid-19---11-march-2020 
2. COVID-19 Map - Johns Hopkins Coronavirus Resource Center [Internet]. Available from: https://coronavirus.jhu.edu/map.html

3. Nunes Duarte-Neto A, de Almeida Monteiro RA, da Silva LFF, et al. Pulmonary and systemic involvement of COVID-19 assessed by ultrasound-guided minimally invasive autopsy [published online ahead of print, 2020 May 22]. Histopathology. 2020;10.1111/his.14160. doi:10.1111/his.14160

4. Mao L, Jin H, Wang M, et al. Neurologic Manifestations of Hospitalized Patients with Coronavirus Disease 2019 in Wuhan, China [published online ahead of print, 2020 Apr 10]. JAMA Neurol. 2020;77(6):1-9. doi:10.1001/jamaneurol.2020.1127

5. Giacomelli A, Pezzati L, Conti F, et al. Self-reported Olfactory and Taste Disorders in Patients With Severe Acute Respiratory Coronavirus 2 Infection: A Cross-sectional Study. Clin Infect Dis. 2020;71(15):889-890. doi:10.1093/cid/ciaa330

6. Herman C, Mayer K, Sarwal A. Scoping review of prevalence of neurologic comorbidities in patients hospitalized for COVID-19. Neurology. 2020;95(2):77-84. doi:10.1212/WNL.0000000000009673

7. Payus AO, Liew Sat Lin C, Mohd Noh M, et al.. SARS-CoV-2 infection of the nervous system: A review of the literature on neurological involvement in novel coronavirus disease(COVID-19). . Bosn J Basic Med Sci. 2020;20(3):283-292. doi:10.17305/bjbms.2020.4860

8. Correia AO, Feitosa PWG, Moreira JLDS, et al. Neurological manifestations of COVID-19 and other coronaviruses: A systematic review. Neurol Psychiatry Brain Res. 2020;37:27-32 doi:10.1016/j.npbr.2020.05.008

9. Finsterer J, Scorza FA, Ghosh R. et al. COVID-19 polyradiculitis in 24 patients without SARS-CoV-2 in the cerebro-spinal fluid [published online ahead of print, 2020 Jun 4]. J Med Virol. 2020;10.1002/jmv.26121. doi:10.1002/jmv.26121

10. Beyrouti R, Adams ME, Benjamin L, et al. Characteristics of ischaemic stroke associated with COVID-19. J Neurol Neurosurg Psychiatry. 2020;91(8):889-891. doi:10.1136/jnnp2020-323586

11. Agosti E, Giorgianni A, D’Amore F, et al. Is Guillain-Barrè syndrome triggered by SARSCoV-2? Case report and literature review [published online ahead of print, $2020 \mathrm{Jul} 9$ ]. Neurol Sci. 2020;1-6. doi:10.1007/s10072-020-04553-9

12. Giorgianni A, Vinacci G, Agosti E, et al. Transient acute-onset tetraparesis in a COVID-19 patient [published online ahead of print, 2020 Jun 2]. Spinal Cord. 2020;1-3. doi:10.1038/s41393-020-0493-8

13. Baracchini C, Pieroni A, Kneihsl M, et al. Practice recommendations for neurovascular 
ultrasound investigations of acute stroke patients in the setting of the COVID-19 pandemic: an expert consensus from the European Society of Neurosonology and Cerebral Hemodynamics [published online ahead of print, 2020 May 19]. Eur J Neurol. 2020;10.1111/ene.14334. doi:10.1111/ene.14334

14. Kaya Y, Kara S, Akinci C, Sagduyu Kocamana A. Transient cortical blindness in COVID-19 pneumonia; a PRES-like syndrome: Case report. J Neurol Sci. 2020;413:116858. doi:10.1016/j.jns.2020.116858

15. Moriguchi T, Harii N, Goto J, et al. A first case of meningitis/encephalitis associated with SARS-Coronavirus-2. Int J Infect Dis. 2020;94:55-58. doi:10.1016/j.ijid.2020.03.062

16. Helms J, Kremer S, Merdji H, et al. Neurologic Features in Severe SARS-CoV-2 Infection. N Engl J Med. 2020;382(23):2268-2270. doi:10.1056/NEJMc2008597

17. Goldberg MF, Goldberg MF. Cerebrovascular Disease in COVID-19. AJNR Am J Neuroradiol. 2020;41(7):1170-1172. doi:10.3174/ajnr.A6588

18. Kandemirli SG, Dogan L, Sarikaya ZT, et al. Brain MRI Findings in Patients in the Intensive Care Unit with COVID-19 Infection [published online ahead of print, 2020 May 8]. Radiology. 2020;201697. doi:10.1148/radiol.2020201697

19. Benussi A, Pilotto A, Premi E, et al. Clinical characteristics and outcomes of inpatients with neurologic disease and COVID-19 in Brescia, Lombardy, Italy [published online ahead of print, 2020 May 22]. Neurology. 2020;10.1212/WNL.0000000000009848. doi:10.1212/WNL.0000000000009848

20. Jahanshahlua L, Rezaei N. Central Nervous System Involvement in COVID-19 [published online ahead of print, 2020 May 22]. Arch Med Res. 2020;S0188-4409(20)30797-9. doi:10.1016/j.arcmed.2020.05.016

21. Kishfy 1, Casasola M, Banankhah P, et al. Posterior reversible encephalopathy syndrome (PRES) as a neurological association in severe Covid-19. J Neurol Sci. 2020;414:116943. doi:10.1016/j.jns.2020.116943

22. Mishra AK, Sahu KK, George AA, Sargent J, Lal A. Cerebrovascular events in COVID-19 patients Monaldi Arch Chest Dis. 2020;90(2):10.4081/monaldi.2020.1341. doi:10.4081/monaldi.2020.1341

23. Kremer S, Lersy F, de Sèze J, et al. Brain MRI Findings in Severe COVID-19: A Retrospective Observational Study [published online ahead of print, 2020 Jun 16]. Radiology. 2020;202222. doi:10.1148/radiol.2020202222

24. Oxley TJ, Mocco J, Majidi S, et al. Large-Vessel Stroke as a Presenting Feature of Covid-19 in the Young. N Engl J Med. 2020 May;382(20):e60 doi:10.1056/NEJMc2009787 
25. Reichard RR, Kashani KB, Boire NA, Constantopoulos E, Guo Y, Lucchinetti CF. Neuropathology of COVID-19: a spectrum of vascular and acute disseminated encephalomyelitis (ADEM)-like pathology. Acta Neuropathol. 2020;140(1):1-6. doi:10.1007/s00401-020-02166-2

26. Radmanesh A, Raz E, Zan E, , et al. Brain Imaging Use and Findings in COVID-19: A Single Academic Center Experience in the Epicenter of Disease in the United States. AJNR Am J Neuroradiol. 2020;41(7):1179-1183. doi:10.3174/ajnr.A6610

27. Giorgianni A, Vinacci G, Agosti E, Mercuri A, Baruzzi F. Neuroradiological features in COVID-19 patients: First evidence in a complex scenario [published online ahead of print, 2020 May 15]. J Neuroradiol. 2020;S0150-9861(20)30169-3. doi:10.1016/j.neurad.2020.05.005

28. Politi LS; Salsano E, Grimaldi M. Magnetic Resonance Imaging Alteration of the Brain in a Patient with Coronavirus Disease 2019 (COVID-19) and Anosmia. [published online ahead of print, 2020 May 29]. JAMA Neurol. 2020;10.1001/jamaneurol.2020.2125. doi:10.1001/jamaneurol.2020.2125

29. Lodigiani C, Iapichino G, Carenzo L, et al. S Venous and arterial thromboembolic complications in COVID-19 patients admitted to an academic hospital in Milan, Italy. Thromb Res. 2020;191:9-14. doi:10.1016/j.thromres.2020.04.024

30. Jain R, Young M, Dogra S, et al. COVID-19 related neuroimaging findings: A signal of thromboembolic complications and a strong prognostic marker of poor patient outcome. $\mathrm{J}$ Neurol Sci. 2020;414:116923. doi:10.1016/j.jns.2020.116923

31. Mahammedi A, Saba L, Vagal A, et al. Imaging in Neurological Disease of Hospitalized COVID-19 Patients: An Italian Multicenter Retrospective Observational Study [published online ahead of print, 2020 May 21]. Radiology. 2020;201933. doi:10.1148/radiol.2020201933

32. Dogra S, Jain R, Cao M, et al. Hemorrhagic stroke and anticoagulation in COVID-19. J Stroke Cerebrovasc Dis. 2020;29(8):104984. doi:10.1016/j.jstrokecerebrovasdis.2020.104984

33. Yaghi S, Ishida K, Torres J, et al. SARS2-CoV-2 and Stroke in a New York Healthcare System. Stroke. 2020;51(7):2002-2011. doi:10.1161/STROKEAHA.120.030335

34. Cantador E, Núñez A, Sobrino P, et al. Incidence and consequences of systemic arterial thrombotic events in COVID-19 patients [published online ahead of print, 2020 Jun 9]. J Thromb Thrombolysis. 2020;1-5. doi:10.1007/s11239-020-02176-7

35. Pons-Escoda A, Naval-Baudín P, Majós C, Camins A, Cardona P, Cos M, Calvo N. 
Neurologic Involvement in COVID-19: Cause or Coincidence? A Neuroimaging Perspective. AJNR Am J Neuroradiol. 2020 Aug;41(8):1365-1369. doi: 10.3174/ajnr.A6627.

36. Li Y, Li M, Wang M, et al. Acute cerebrovascular disease following COVID-19: a single center, retrospective, observational study [published online ahead of print, $2020 \mathrm{Jul}$ 2]. Stroke Vasc Neurol. 2020;svn-2020-000431. doi:10.1136/svn-2020-000431

37. Nalleballe K, Reddy Onteddu S, Sharma R, et al. Spectrum of neuropsychiatric manifestations in COVID-19. Brain Behav Immun. 2020 Aug;88:71-74. doi: 10.1016/j.bbi.2020.06.020.

38. Merkler AE, Parikh NS, Mir S, et al. Risk of Ischemic Stroke in Patients With Coronavirus Disease 2019 (COVID-19) vs Patients With Influenza [published online ahead of print, 2020 Jul 2]. JAMA Neurol. 2020;e202730. doi:10.1001/jamaneurol.2020.2730

39. Rothstein A, Oldridge O, Schwennesen H, Do D, Cucchiara BL. Acute Cerebrovascular Events in Hospitalized COVID-19 Patients. Stroke. 2020 Sep;51(9):e219-e222. doi: 10.1161/STROKEAHA.120.030995.

40. Altschul DJ, Unda SR, de La Garza Ramos R, et al. Hemorrhagic presentations of COVID19: Risk factors for mortality. Clin Neurol Neurosurg. 2020;198:106112. doi:10.1016/j.clineuro.2020.106112

41. Requena M, Olivé-Gadea M, Muchada M, et al. COVID-19 and Stroke: Incidence and Etiological Description in a High-Volume Center. J Stroke Cerebrovasc Dis. 2020;29(11):105225. doi:10.1016/j.jstrokecerebrovasdis.2020.105225

42. Katz JM, Libman RB, Wang JJ, et al. Cerebrovascular Complications of COVID-19. Stroke. 2020 Sep;51(9):e227-e231. doi: 10.1161/STROKEAHA.120.031265. Epub 2020 Aug 6.

43. Shahjouei S, Naderi S, Li J, Khan A, et al. Risk of stroke in hospitalized SARS-CoV-2 infected patients: A multinational study. EBioMedicine. 2020 Sep;59:102939. doi: 10.1016/j.ebiom.2020.102939.

44. Iltaf S Sr, Fatima M, Salman S Sr, Salam JU, Abbas S. Frequency of Neurological Presentations of Coronavirus Disease in Patients Presenting to a Tertiary Care Hospital During the 2019 Coronavirus Disease Pandemic. Cureus. 2020 Aug 18;12(8):e9846. doi: $10.7759 /$ cureus.9846

45. Romero-Sánchez CM, Díaz-Maroto I, Fernández-Díaz E, et al. Neurologic manifestations in hospitalized patients with COVID-19: The ALBACOVID registry. Neurology. 2020 Aug 25;95(8):e1060-e1070. doi: 10.1212/WNL.0000000000009937.

46. Xiong W, Mu J, Guo J, et al. New onset neurologic events in people with COVID-19 in 3 regions in China. Neurology. 2020 Sep 15;95(11):e1479-e1487. doi: 


\subsection{2/WNL.0000000000010034.}

47. Siegler JE, Cardona P, Arenillas JF, et al. Cerebrovascular events and outcomes in hospitalized patients with COVID-19: The SVIN COVID-19 Multinational Registry. Int J Stroke. 2020 Sep 30:1747493020959216. doi: 10.1177/1747493020959216.

48. Rifino N, Censori B, Agazzi E, et al. Neurologic manifestations in 1760 COVID-19 patients admitted to Papa Giovanni XXIII Hospital, Bergamo, Italy. J Neurol. 2020 Oct 7:1-8. doi: 10.1007/s00415-020-10251-5.

49. Sabayan B, Moghadami M, Assarzadegan F, et al. COVID-19 Respiratory Illness and Subsequent Cerebrovascular Events, the Initial Iranian Experience. J Stroke Cerebrovasc Dis. 2021 Jan;30(1):105454. doi: 10.1016/j.jstrokecerebrovasdis.2020.105454.

50. Lee E, Krajewski A, Clarke C, O'Sullivan D, Herbst T, Lee S. Arterial and venous thromboembolic complications of COVID-19 detected by CT angiogram and venous duplex ultrasound. Emerg Radiol. 2021 Jan 11:1-8. doi: 10.1007/s10140-020-01884-0.

51. Baig AM, Khaleeq A, Ali U, Syeda H. Evidence of the COVID-19 Virus Targeting the CNS: Tissue Distribution, Host-Virus Interaction, and Proposed Neurotropic Mechanisms. ACS Chem Neurosci. 2020;11(7):995-998. doi:10.1021/acschemneuro.0c00122

52. Varga Z, Flammer AJ, Steiger P, et al. Endothelial cell infection and endotheliitis in COVID19. Lancet. 2020;395(10234):1417-1418. doi:10.1016/S0140-6736(20)30937-5

53. Mehta P, McAuley DF, Brown M, Sanchez E, Tattersall RS, Manson JJ. COVID-19: consider cytokine storm syndromes and immunosuppression. Lancet. 2020;395(10229):1033-4 doi:10.1016/S0140-6736(20)30628-0

54. Spiezia L, Boscolo A, Poletto F, et al. COVID-19-Related Severe Hypercoagulability in Patients Admitted to Intensive Care Unit for Acute Respiratory Failure. Thromb Haemost. 2020;120(6):998-1000. doi:10.1055/s-0040-1710018

55. Zhang Y, Xiao M, Zhang S, et al. Coagulopathy and Antiphospholipid Antibodies in Patients with Covid-19. N Engl J Med. 2020;382(17):e38. doi:10.1056/NEJMc2007575

56. Bhatla A, Mayer MM, Adusumalli S, et al. COVID-19 and cardiac arrhythmias. Heart Rhythm. 2020;17(9):1439-1444. doi:10.1016/j.hrthm.2020.06.016

57. Kochi AN, Tagliari AP, Forleo GB, Fassini GM, Tondo C. Cardiac and arrhythmic complications in patients with COVID-19. J Cardiovasc Electrophysiol. 2020 May;31(5):1003-1008. doi: 10.1111/jce.14479.

58. Aghagoli G, Gallo Marin B, Soliman LB, Sellke FW. Cardiac involvement in COVID-19 patients: Risk factors, predictors, and complications: A review. J Card Surg. 2020 Jun;35(6):1302-1305. doi: 10.1111/jocs.14538. 
59. Boehme AK, Luna J, Kulick ER, Kamel H, Elkind MSV. Influenza-like illness as a trigger for ischemic stroke. Ann Clin Transl Neurol. 2018;5(4):456-463. Published 2018 Mar 14. doi:10.1002/acn3.545

60. Montalvan V, Lee J, Bueso T, De Toledo J, Rivas K. Neurological manifestations of COVID-19 and other coronavirus infections: A systematic review. Clin Neurol Neurosurg. 2020;194:105921. doi:10.1016/j.clineuro.2020.105921

61. Wu Y, Xu X, Chen Z, et al. Nervous system involvement after infection with COVID-19 and other coronaviruses. Brain Behav Immun. 2020;87:18-22. doi:10.1016/j.bbi.2020.03.031

62. Parauda SC, Gao V, Gewirtz AN, et al. Posterior reversible encephalopathy syndrome in patients with COVID-19 [published online ahead of print, 2020 Jul 9]. J Neurol Sci. 2020;416:117019. doi:10.1016/j.jns.2020.117019

63. Conte G, Avignone S, Carbonara M, et al. COVID-19 - Associated PRES - like Encephalopathy with Perivascular Gadolinium Enhancement. [published online ahead of print, 2020 Aug 13]. AJNR Am J Neuroradiol. 2020; 10.3174/ajnr.A6762 doi:10.3174/ajnr.A6762

64. D'Amore F, Vinacci G, Agosti E, et al. Pressing Issues in COVID-19: Probable Cause to Seize SARS-CoV-2 for Its Preferential Involvement of Posterior Circulation Manifesting as Severe Posterior Reversible Encephalopathy Syndrome and Posterior Strokes [published online ahead of print, 2020 Jul 30]. AJNR Am J Neuroradiol. 2020;10.3174/ajnr.A6679. doi:10.3174/ajnr.A6679

65. Franceschi AM, Ahmed O, Giliberto L, Castillo M. Hemorrhagic Posterior Reversible Encephalopathy Syndrome as a Manifestation of COVID-19 Infection. AJNR Am J Neuroradiol. 2020;41(7):1173-1176. doi:10.3174/ajnr.A6595

66. Gao B, Lyu C, Lerner A, McKinney AM. Controversy of posterior reversible encephalopathy syndrome: what have we learnt in the last 20 years?. J Neurol Neurosurg Psychiatry. 2018;89(1):14-20. doi:10.1136/jnnp-2017-316225

67. Cagnazzo F, Arquizan C, Derraz I, Dargazanli C, Lefevre PH, Riquelme C, Gaillard N, Mourand I, Gascou G, Bonafe A, Costalat V. Neurological manifestations of patients infected with the SARS-CoV-2: a systematic review of the literature. J Neurol. 2020 Oct 30:1-10. doi: 10.1007/s00415-020-10285-9.

68. Italian Stroke Organization. Italian Stroke Organization Guidelines [Internet]. 2016. Available from: https://www.iso-stroke.it/wp-content/uploads/2017/02/LIBRO-SPREADVIII-ED-13-09-16.pdf

69. Nemoto W, Yamagata R, Nakagawasai O, Nakagawa K, Hung WY, Fujita M, et al. Effect of 
spinal angiotensin-converting enzyme 2 activation on the formalin-induced nociceptive response in mice. Eur J Pharmacol. 2020;

70. Munz M, Wessendorf S, Koretsis G, Tewald F, Baegi R, Krämer S, et al. Acute transverse myelitis after COVID-19 pneumonia. Journal of Neurology. 2020.

71. Scheidl E, Canseco DD, Hadji-Naumov A, Bereznai B. Guillain-Barré syndrome during SARS-CoV-2 pandemic: A case report and review of recent literature. J Peripher Nerv Syst. 2020;25(2):204-207. doi:10.1111/jns.12382

72. Koralnik IJ, Tyler KL. COVID-19: A Global Threat to the Nervous System. Ann Neurol. 2020;88(1):1-11. doi:10.1002/ana.25807

73. Palao M, Fernández-Díaz E, Gracia-Gil J, Romero-Sánchez CM, Díaz-Maroto I, Segura T. Multiple sclerosis following SARS-CoV-2 infection. Mult Scler Relat Disord. 2020;45:102377. [published online ahead of print, 2020 Aug 13] doi:10.1016/j.msard.2020.102377

74. Sadeghmousavi S, Rezaei N. COVID-19 and Multiple Sclerosis: Predisposition and Precautions in Treatment. SN Compr Clin Med. 2020 Sep 3:1-6. doi: 10.1007/s42399-02000504-9. 


\section{Table 1}

\section{Demographic and clinical data}

$\mathrm{AF}=$ atrial fibrillation, $\mathrm{CAD}=$ coronary artery disease, $\mathrm{COPD}=$ chronic obstructive pulmonary disease $\mathrm{CKD}=$ chronic kidney disease, $\mathrm{ICH}=$ intracerebral hemorrhage, $\mathrm{SAH}=$ subarachnoid hemorrhage, PRES = posterior reversible encephalopathy syndrome, $\mathrm{SDH}=$ subdural hematoma.

\section{Table 2}

\section{Neuroimaging studies}

$\mathrm{ICH}=$ intracerebral hemorrhage, $\mathrm{SAH}=$ subarachnoid hemorgrhage, $\mathrm{PRES}=$ posterior reversible encephalopathy syndrome, $\mathrm{a} / \mathrm{c} \mathrm{SDH}=$ acute on chronic subdural hemorrhage

\section{Table 3}

\section{Neuroimaging findings}

$\mathrm{ICH}=$ intracerebral hemorrhage, $\mathrm{SAH}=$ subarachnoid hemorrhage, PRES $=$ posterior reversible encephalopathy syndrome, $\mathrm{a} / \mathrm{c} \mathrm{SDH}=$ acute on chronic subdural hemorrhage, $\mathrm{MS}=$ multiple sclerosis, $\mathrm{ADEM}=$ acute disseminated encephalomyelitis, GBS = Guillain-Barré Syndrome

\section{Table 4}

\section{Diseases prevalence and demographic data in the study cohort.}

$\mathrm{CVD}=$ cerebrovascular disease, $\mathrm{AIS}=$ acute ischemic stroke, $\mathrm{ICH}=$ intracerebral hemorrhage, $\mathrm{SAH}$ = subarachnoid hemorrhage, $\mathrm{PRES}=$ posterior reversible encephalopathy syndrome, $\mathrm{a} / \mathrm{c} \mathrm{SDH}=$ acute on chronic subdural hemorrhage.

\section{Table 5}

Prevalence and demographic data of cerebrovascular diseases in COVID-19 patients.

AIS = acute ischemic stroke, $\mathrm{ICH}=$ intracranial hemorrhage, $\mathrm{PRES}=$ posterior reversible encephalopathy syndrome

\section{Figure 1}

Flowchart of the multicenter study. 


\section{Figure 2}

Anterior circulation stroke. A 85 year-old woman with history of hypertension presenting with sudden onset of left hemiparesis. (A) CT without contrast shows an ill-defined cortical-subcortical hypoattenuating area in the right lateral posterior frontal lobe. (B) CTA VR reformat shows thrombotic occlusion of the (right) middle cerebral artery M2 segment. (C-D) b1000 and ADC map: the lesion shows restricted diffusion and (E) T2-FLAIR hyperintensity consistent with acute ischemia.

\section{Figure 3}

Dural sinus thrombosis and venous ischemia. A 61 year-old woman with history of hypertension presenting with altered consciousness and headache. CT without contrast shows hyperattenuating right transverse sinus (a) straight sinus, vein of Galen and internal cerebral veins (b) and focal mesial parietal ischemic changes (c).Venous CT angiography MIP reformats show filling defects at the level of the right transverse sinus and torcula Herophilii (d), jugular bulb and superior sagittal sinus (e) sinus and right internal cerebral vein (f). A somewhat generalized superficial venous engorgement is also noted.

\section{Figure 4}

Hemorrhagic stroke. A 79 year-old male with history of hypertension with loss of consciousness and coma. Upon admission CT without contrast (A and B) shows right frontal-parietal hematoma with massive intraventricular extension.

\section{Figure 5}

PRISMA-ScR Extension flow diagram. 
Figures

7937 consecutive confirmed COVID-19 patients admitted from February 21 to July 10

$253 / 7937$ (3\%) patients had abnormal neurological examination who underwent neuroimaging

90/253 (36\%) patients showed acute neuroimaging findings:

- $65(72 \%)$ ischemic strokes

- 1 venous infarct

- $12(13 \%)$ intracranial hemorrhages

- $3(3 \%)$ PRES

- $6(7 \%)$ encephalitides

- $4(4 \%)$ demyelinating diseases

- 1 MS exacerbation

- 1 new onset MS

- 1 ADEM

- $1 \mathrm{GBS}$

Figure 1

Flowchart of the multicenter study. 

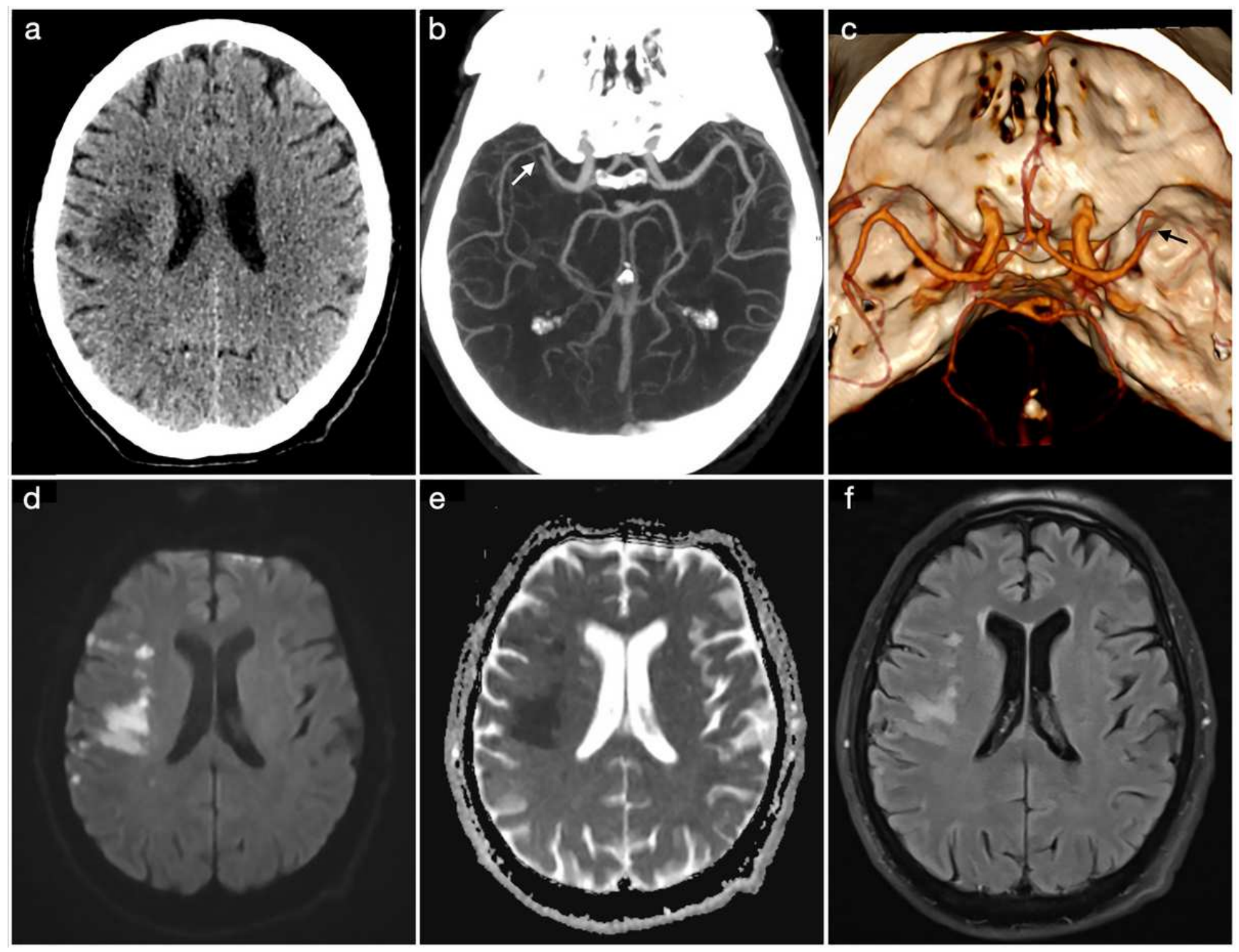

Figure 2

Anterior circulation stroke. A 85 year-old woman with history of hypertension presenting with sudden onset of left hemiparesis. (A) CT without contrast shows an ill-defined cortical-subcortical hypoattenuating area in the right lateral posterior frontal lobe. (B) CTA VR reformat shows thrombotic occlusion of the (right) middle cerebral artery M2 segment. (C-D) b1000 and ADC map: the lesion shows restricted diffusion and (E) T2-FLAIR hyperintensity consistent with acute ischemia. 


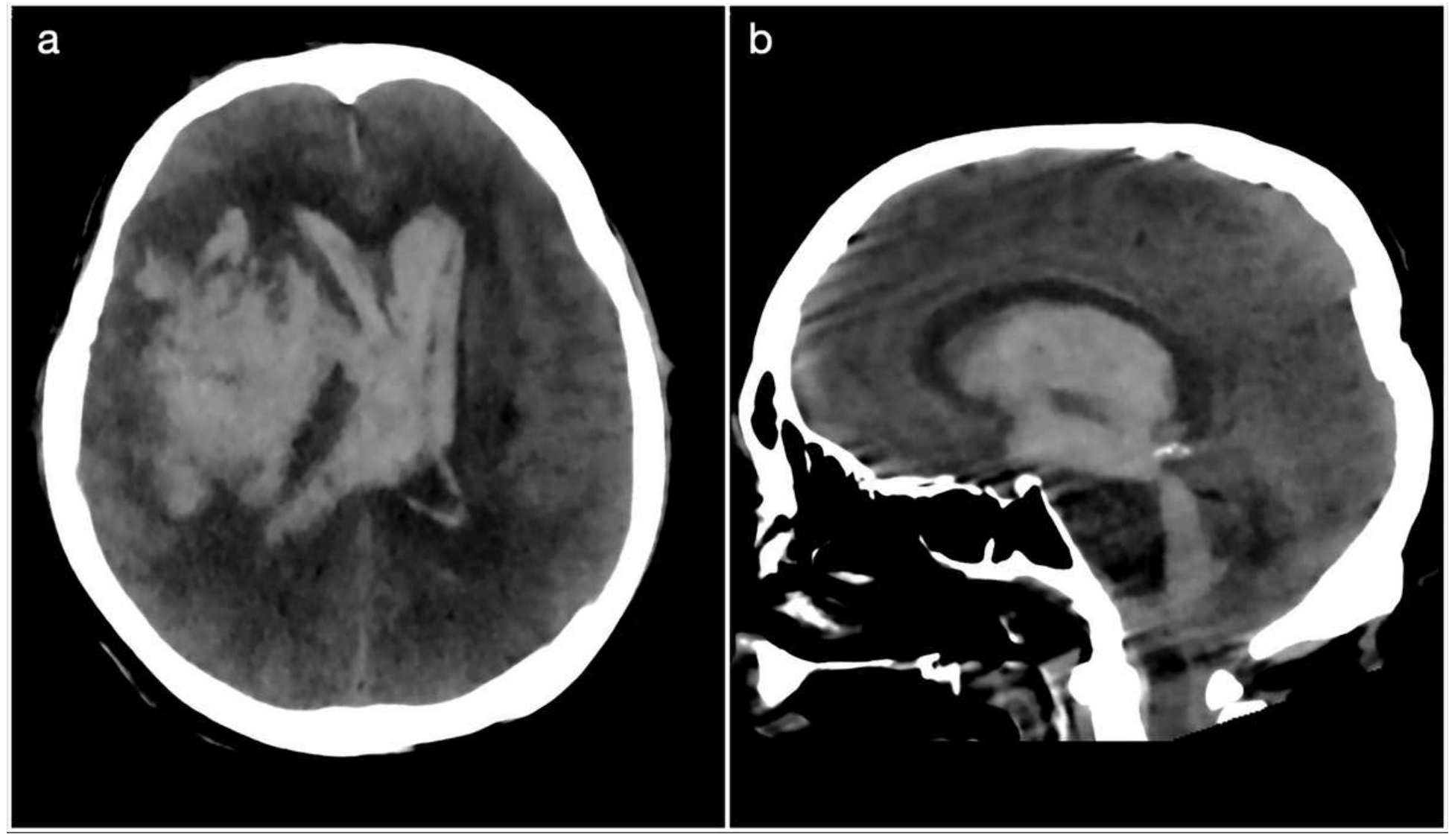

\section{Figure 3}

Dural sinus thrombosis and venous ischemia. A 61 year-old woman with history of hypertension presenting with altered consciousness and headache. CT without contrast shows hyperattenuating right transverse sinus (a) straight sinus, vein of Galen and internal cerebral veins (b) and focal mesial parietal ischemic changes (c).Venous CT angiography MIP reformats show filling defects at the level of the right transverse sinus and torcula Herophilii (d), jugular bulb and superior sagittal sinus (e) sinus and right internal cerebral vein (f). A somewhat generalized superficial venous engorgement is also noted. 

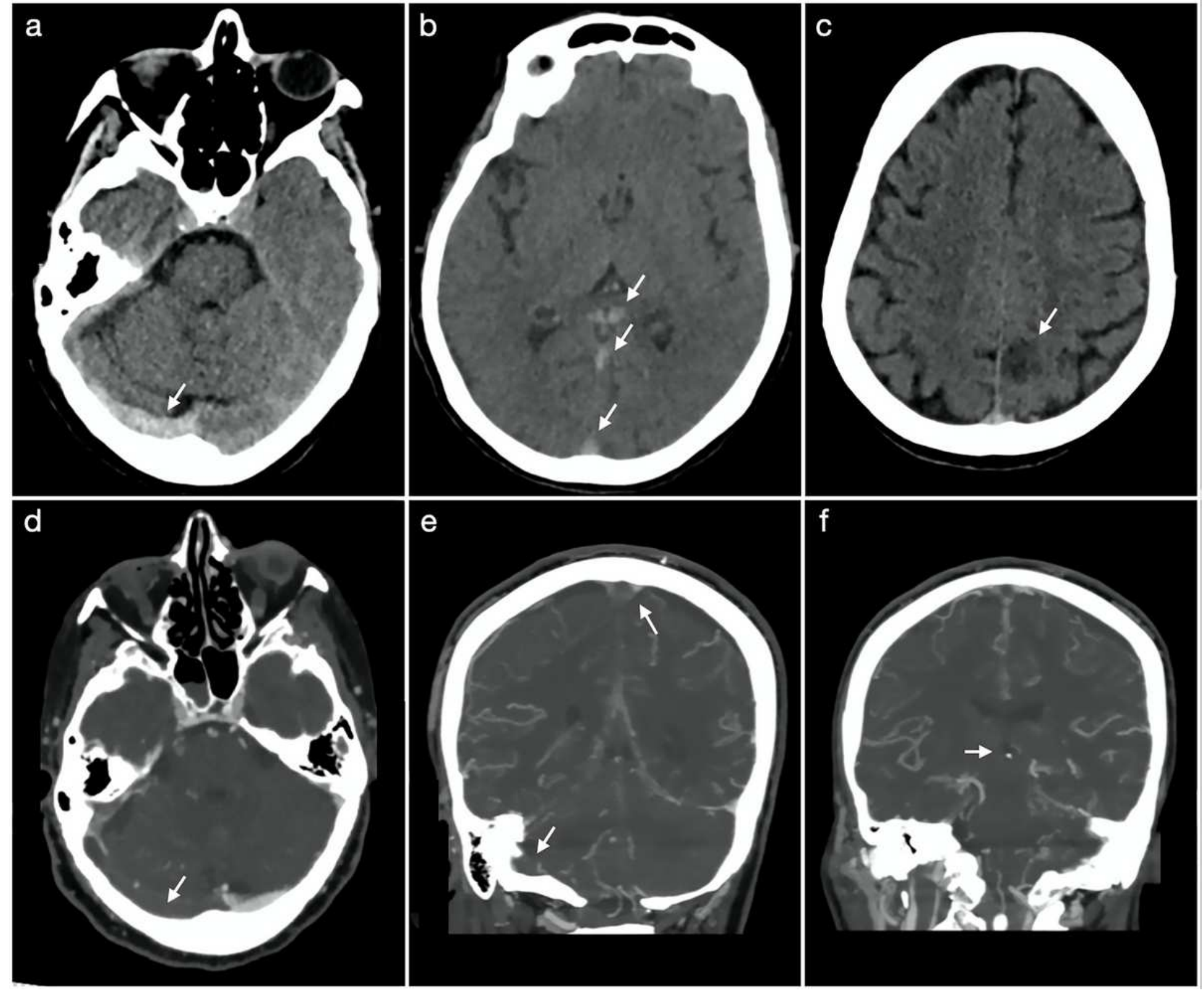

Figure 4

Hemorrhagic stroke. A 79 year-old male with history of hypertension with loss of consciousness and coma. Upon admission CT without contrast (A and B) shows right frontal-parietal hematoma with massive intraventricular extension. 


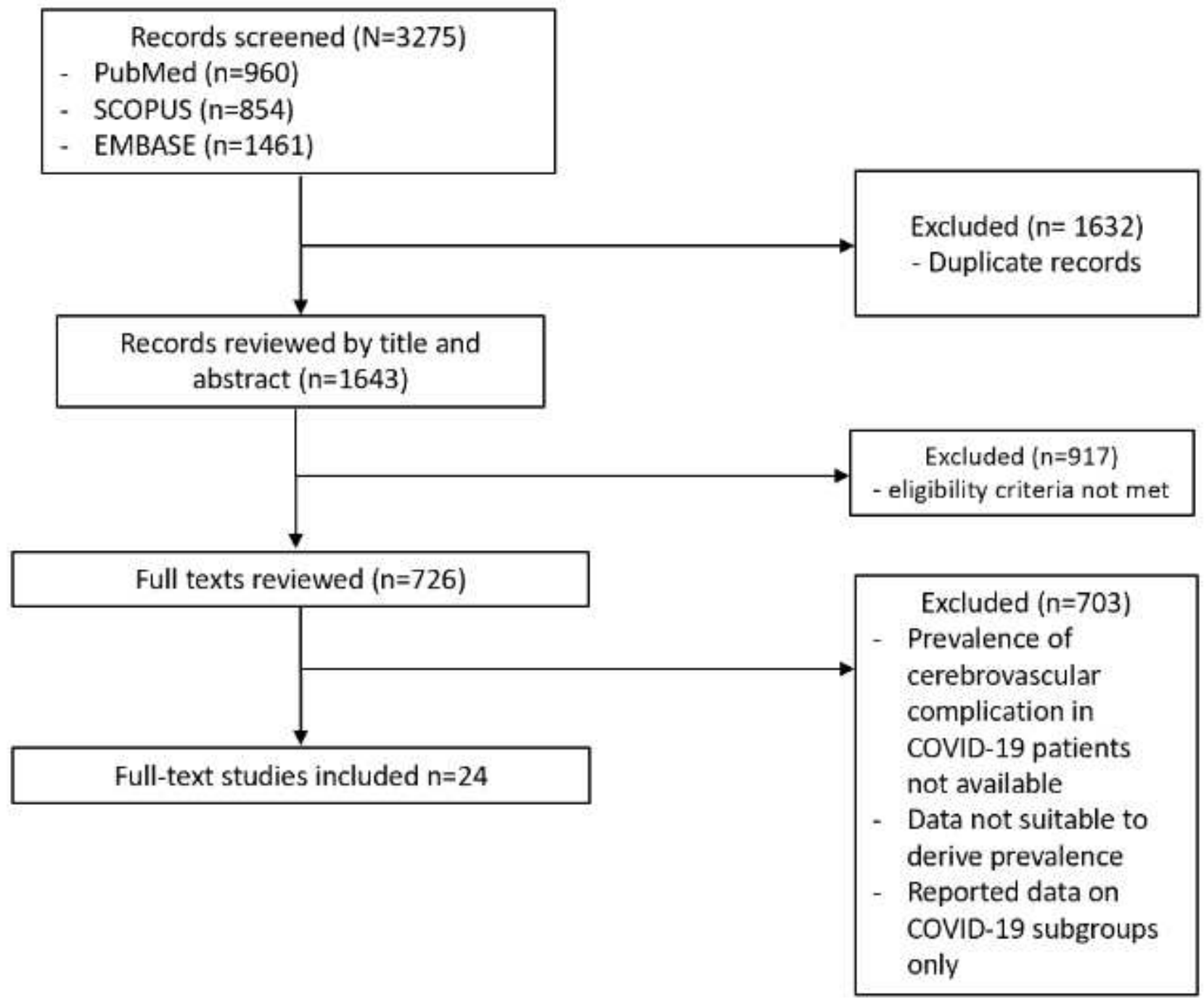

Figure 5

PRISMA-ScR Extension flow diagram. 